\title{
Wavelength Evolution of Fiber Bragg Gratings Fabricated From Hydrogen-Loaded Optical Fiber During Annealing
}

Yuji Masuda, Mitsuo Nakamura, Chisa Komatsu, Keio Fujita, Makoto Yamauchi, Masanori Kimura, Yasuo Mizutani, Susumu Kimura, Yoshifumi Suzaki, Member, IEEE, Takashi Yokouchi, Kiyoshi Nakagawa, Senior Member, IEEE, and Seiki Ejima

\begin{abstract}
The shift in the central wavelength of fiber Bragg gratings (FBGs) during annealing of hydrogen-loaded optical fiber exposed to ultraviolet (UV) laser irradiation through a phase mask is studied and shown to be caused by thermal diffusion of hydrogen out of the fiber for both short-period and long-period FBGs. Reloading FBGs with hydrogen followed by a second annealing cycle without exposing the fiber to the UV laser reproduces the details of the wavelength evolution observed during first annealing following UV irradiation. This shows that the wavelength shift of the grating during annealing is determined by diffusion of hydrogen gas out of the optical fiber for both short-period and long-period FBGs.
\end{abstract}

Index Terms-Annealing, Bragg grating, diffusion, excimer laser, hydrogen, long-period fiber Bragg grating (FBG), optical fiber, short-period fiber Bragg grating (FBG), silica, ultraviolet (UV) photosensitivity.

\section{INTRODUCTION}

$\mathbf{I}$ T has been noticed that the central wavelength of fiber Bragg gratings (FBGs) fabricated by exposing hydrogen loaded optical fiber to ultraviolet (UV) laser irradiation drifts to shorter wavelengths by about $0.5-1 \mathrm{~nm}$ over a period ranging from a few to several weeks when it is not heat-treated [1]. This wavelength shift exceeds or is comparable to the channel separation employed in telecommunications systems, about 0.78 or $0.39 \mathrm{~nm}$ for 100 - and $50-\mathrm{GHz}$ dense-wavelength-division-multiplexing (DWDM) systems, respectively. The wavelength can be stabilized if the FBG is processed by annealing. However, design becomes more complicated because a wavelength offset must be included. The wavelength shift is problematic not only for telecom applications, but also for other applications, and good control is needed. In long-period FBGs (LP-FBG), the shift is about two orders of magnitude greater than in short-period FBGs (SP-FBG) and can be as much as several tens of

Manuscript received August 25, 2003; revised December 2, 2003.

Y. Masuda, K. Fujita, Y. Suzaki, T. Yokouchi, K. Nakagawa, and S. Ejima are with Faculty of Engineering, Kagawa University, 2217-20 Kagawa, Japan (e-mail: ejima@eng.kagawa-u.ac.jp).

M. Nakamura was with the Faculty of Engineering, Kagawa University, 2217-20 Kagawa, Japan. He is now with the Graduate School of Engineering, Osaka University, Osaka 565-0871, Japan.

C. Komatsu was with the Faculty of Engineering, Kagawa University, 2217-20 Kagawa, Japan. She is now with Fujitsu Kochi Systems Engineering, Ltd., Kochi, Japan.

M. Yamauchi, M. Kimura, Y. Mizutani, and S. Kimura are with Shinko Electric Wire Company, Ltd., 1298-12 Shido, Kagawa, Japan.

Digital Object Identifier 10.1109/JLT.2004.825784 nanometers. In order to make use of the excellent characteristics of FBGs, in particular their sharp spectral response, we need to understand the mechanism of the wavelength shift and to be able to control it accurately.

The wavelength shift certainly depends on the processes used to fabricate an FBG. Numerous studies have been made of the origin of the UV photosensitivity, including the color center model [2], photoelastic model [3], [4], and so forth. These theories are quite complex, and it appears that the photosensitivity may be determined by several different mechanisms, rather than by a single mechanism.

Before an optical fiber is exposed to a UV laser, it is usually loaded with hydrogen gas in order to increase its UV photosensitivity [5], and it is annealed after imprinting an FBG to stabilize its reflectivity and central wavelength. For the annealing process, Erdogan [6] has developed an empirical theory to describe how the reflectivity of the FBG decreases during annealing for fibers without hydrogen loading in accordance with a power law model based on electron detrapping by annealing from broadly distributed traps which capture electrons by UV absorption. Baker et al. [7] have shown that for hydrogen-loaded fibers, the spectral evolution deviates from Erdogan's power law model, and they have proposed a log-time model. The deviation appears to take place in the early stage of annealing. Erdogan's theory has been extended to other fibers in the search for thermally stable FBGs, such as boron-doped fibers, tin-doped fibers, and fluorine-doped fibers [8]-[10]. The fibers used in these studies were without hydrogen loading or such that hydrogen gas was completely removed by leaving them for several days at ambient temperature. There, the central wavelength varies by $0.1-0.2 \mathrm{~nm}$ or less.

For fibers loaded with hydrogen, the central wavelength of an FBG without annealing has been reported to evolve gradually to shorter wavelengths by about $0.7 \mathrm{~nm}$, much more than for those without hydrogen at room temperature, owing to out-diffusion of hydrogen from the fiber over a long time, ranging from a few to several hundred hours [1], [11].

Malo et al. [1] have interpreted their results in terms of hydrogen molecules that are dissociated by UV irradiation and react with dopant $\mathrm{Ge}$ to form $\mathrm{Ge}-\mathrm{OH}$ bonds in the fiber core; hence, the hydrogen molecules are depleted there. In the cladding, where Ge is absent, the dissociated hydrogen simply recombines and refills the fiber core by inward diffusion. This in-diffusion increases the central wavelength for several hours 
after UV exposure. After that, outward diffusion from the bulk causes a decrease in the central wavelength over a few to several hundred hours at room temperature. Thus, the central wavelength initially increases and then decreases. They have further shown that, when the FBG is reloaded with hydrogen gas, the central wavelength simply decreases without showing the upswing, confirming the absence of hydrogen depletion and in-diffusion without UV exposure.

Swart et al. [12], [13] have done an experiment tracking the change in the refractive index based on interferometry with two FBGs in high-pressure hydrogen up to $100 \mathrm{~atm}$ at $40-90^{\circ} \mathrm{C}$. By loading hydrogen gas and evacuating the gas, they have determined the diffusivity and solubility of hydrogen gas molecules into and out of the fiber silica glass.

Bakhti et al. [14] have studied the wavelength evolution of LP-FBGs at room temperature in a fashion similar to that of Malo et al. [1] for SP-FBGs. After UV exposure, hydrogen out-diffusion from the cladding initially decreases the refractive index for higher order cladding modes, which increases the central wavelength, and later the out-diffusion from the bulk reduces the refractive index of $\mathrm{LP}_{01}$ mode, thereby causing an upswing in the central wavelength similar to that in SP-FBGs.

Increasing evidence exists that the wavelength shift is caused by the diffusion of hydrogen out of the fiber. In order to obtain definitive evidence, we have performed a series of experiments on the evolution of the wavelength during the annealing process at several different annealing temperatures for both short-period and long-period FBGs. The data for both SP-FBGs and LP-FBGs agree well with theoretical predictions for different annealing temperatures and for different hydrogen-loading pressures. To the best of the authors' knowledge, no such combined observations have been reported previously for SP-FBG and LP-FBG. We have also determined the minimum annealing time for different annealing temperatures required to stabilize the central wavelength of FBGs. In order to confirm that the evolution is due to hydrogen out-diffusion, we have reloaded an FBG with hydrogen and annealed it a second time. Here, the evolution of the central wavelength was essentially the same as that observed following the original first annealing; hence, the wavelength shift is certainly controlled by hydrogen out-diffusion. These data are used to determine the change in the refractive index of the optical fiber owing to hydrogen loading.

\section{EXPERIMENTS}

In this experiment, we loaded hydrogen gas into a single-mode optical fiber (Corning SMF-28) at 100-atm pressure and room temperature for ten days and stored it in a freezer at about $-50{ }^{\circ} \mathrm{C}$ for one day to two weeks before using it. We fabricated an SP-FBG by removing the coating resin and exposing the bare fiber to a $\mathrm{KrF} 248-\mathrm{nm}$ excimer laser through a phase mask. The laser fluence is $280 \mathrm{~mJ} / \mathrm{cm}^{2} /$ pulse at the position of the fiber, and the laser was run at a repetition rate of $20 \mathrm{~Hz}$ for $2.5 \mathrm{~min}$. The grating period $p$ of the phase mask is $1073.63 \mathrm{~nm}$, and the effective-refractive index $n_{\text {eff }}$ of the fiber is about 1.446. The central wavelength of the SP-FBG is expected to be $\lambda=2 n_{\text {eff }}(p / 2)=2 n_{\text {eff }} \Lambda \sim 1552.5 \mathrm{~nm}$, where $\Lambda$ is grating pitch of the SP-FBG. The length of the

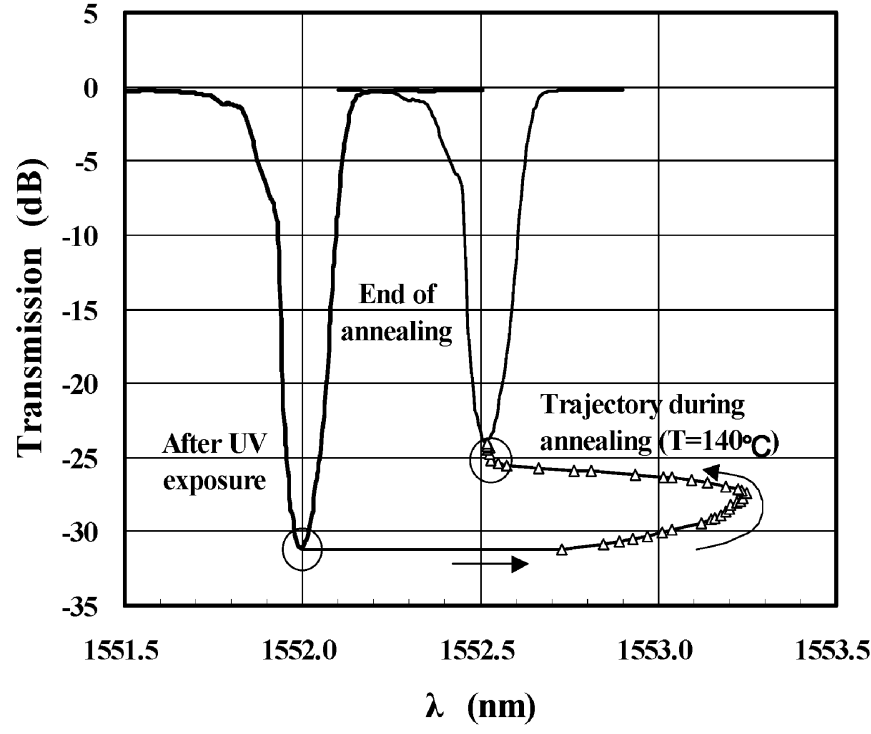

Fig. 1. Evolution of the transmission spectrum of an SP-FBG during annealing.

FBG is $15.5 \mathrm{~mm}$. A weak tension of $\sim 80 \mathrm{gf}$ was applied to the optical fiber for secure mounting during laser irradiation [15].

An LP-FBG was made by exposing the same $\mathrm{H}_{2}$-loaded fiber to the same $\mathrm{KrF}$ excimer laser through multiple slits in thin stainless steel with $50 \%$ opening factor and a $540-\mu \mathrm{m}$ period. The laser irradiation conditions were the same as for SP-FBG fabrication, i.e., $280 \mathrm{~mJ} / \mathrm{cm}^{2} /$ pulse at the position of the fiber and $20-\mathrm{Hz}$ repetition rate. The fiber holder, together with the multiple slits, was moved against the laser beam in the longitudinal direction of the fiber. The beam spot size was $16 \mathrm{~mm}$ in diameter, and the total length of the LP-FBG was $40 \mathrm{~mm}$. Each slit was irradiated by the laser for a total of $45 \mathrm{~s}$.

The fabricated FBGs were placed in an annealing oven and splice-connected to an in situ monitoring optical circuit within $\sim 10$ min after exposure to the laser. The oven was set at the experimental annealing temperature beforehand. We define the start time $t=0$ in the data below as the time when the UV laser irradiation was completed. The spectral evolution of the signal transmitted through the FBG was monitored for about $100 \mathrm{~h}$ by injecting the wide-band signal light from an amplified spontaneous emission (ASE) source.

\section{A. Wavelength Evolution of SP-FBG}

Fig. 1 shows spectra of the light transmitted through the SP-FBG at the end of the UV exposure $(t=0)$ and after $80 \mathrm{~h}$ of annealing at $140{ }^{\circ} \mathrm{C}$. The trajectory of the transmission minimum is also shown there. The initial central wavelength is $1552.0 \mathrm{~nm}$, slightly shorter than that expected from the relation $\lambda=2 n_{\text {eff }}(p / 2)=2 n_{\text {eff }} \Lambda \sim 1552.5 \mathrm{~nm}$. The central wavelength reaches its maximum value after $\sim 15 \mathrm{~min}$ and decreases to a stable value after $\sim 6 \mathrm{~h}$.

The time evolutions of the transmission minimum and peak central wavelength are shown in Fig. 2(a) and (b), respectively, for annealing temperatures $50,100,140$, and $200^{\circ} \mathrm{C}$. The shift in the central wavelength $\Delta \lambda$ is taken to be the difference from a reference that is taken just after the FBG is placed in the oven. 
(a)

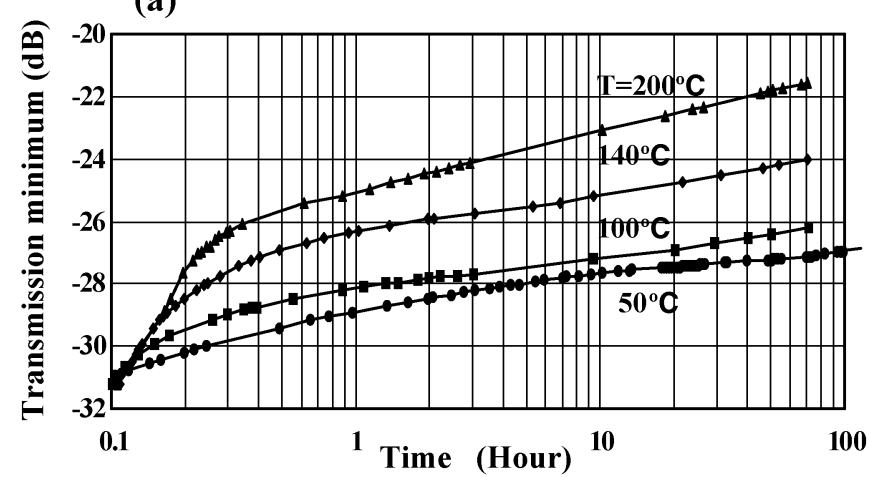

(b)

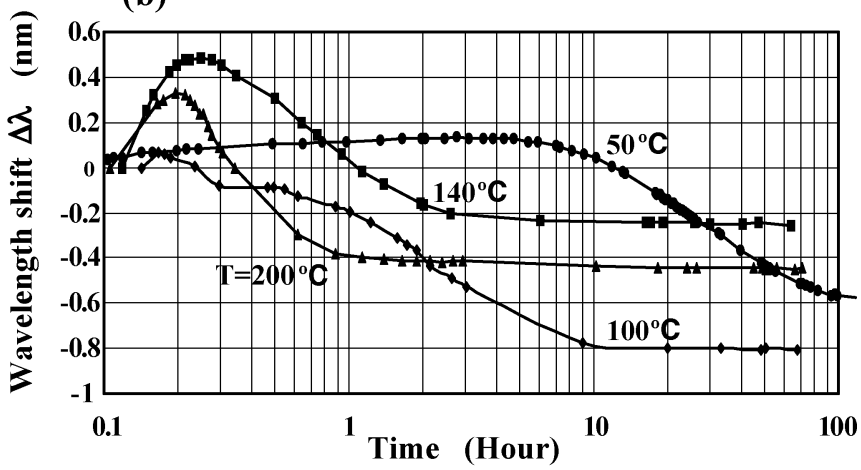

Fig. 2. (a) Transmission minimum and (b) change in the central wavelength of an SP-FBG during annealing.

The $\Delta \lambda$ approaches a stable value after a certain annealing time, but the transmission minimum continues to decrease.

The temperature of the annealing oven was set before the FBG was placed in it but does drop temporarily because the door is opened; however, the temperature recovers after about $15 \mathrm{~min}$, according to thermocouple measurements. The upswing of the central wavelength in Fig. 2(b) is partly due to the temperature dependence of the shift in the FBG wavelength, which is about $0.01 \mathrm{~nm} /{ }^{\circ} \mathrm{C}$. It also appears to be partly due to the hydrogen depletion by UV laser irradiation followed by in-diffusion as described in [1].

\section{B. Wavelength Evolution of $L P-F B G$}

The time evolution of the central wavelength of the LP-FBG is shown in Fig. 3. Normally an LP-FBG manifests several spectral modes. We have tracked the $\mathrm{LP}_{05}$ mode. The mode number is determined in the following way. By fabricating many LP-FBGs with different slit periods and monitoring their spectra through injecting a wide-band signal, $1450 \sim 1610 \mathrm{~nm}$, from the ASE light source (Thorlabs ASE-7701-AP), we have identified the spectrum of the shortest wavelength mode as $\mathrm{LP}_{02}$ in accordance with Fig. 1 of [16] and have confirmed that the mode we have tracked is $\mathrm{LP}_{05}$. The slit period was varied in intervals of $30 \sim 50 \mu \mathrm{m}$ over the range of $540 \sim 690 \mu \mathrm{m}$ using two sets of multiple slits with 540- and $600-\mu$ m periods and additional periods were obtained by tilting these two slits.

The central wavelength of the LP-FBG was $1530 \mathrm{~nm}$ for a slit period of $540 \mu \mathrm{m}$. Unlike the SP-FBG, the transmission minimum was very sensitive to how the fiber is mounted in the annealing oven. The behavior of the central wavelength,

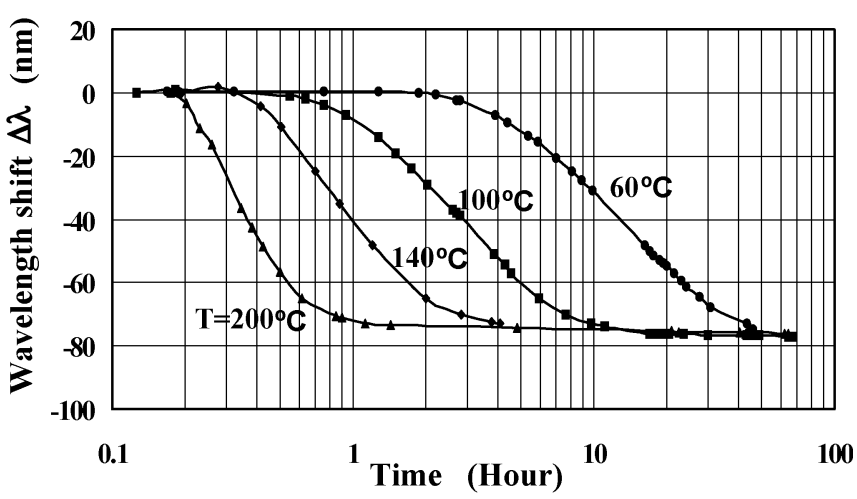

Fig. 3. Change in the central wavelength of an LP-FBG during annealing.

however, was reproducible and is shown in Fig. 3, where the shift is defined as $\Delta \lambda$, the difference from the reference taken immediately after the FBG was placed in the oven. The LP-FBG has a wavelength shift $\Delta \lambda \sim 77 \mathrm{~nm}$, or about two orders of magnitude greater than that for the SP-FBG $(\Delta \lambda \sim 0.7 \mathrm{~nm})$.

\section{Comparison With the $\mathrm{H}_{2}$ Diffusion Theory}

The diffusion of molecular hydrogen is described by the diffusion equation

$$
\frac{\partial C}{\partial t}-D \nabla^{2} C=0
$$

where $C$ is the hydrogen concentration. The diffusion coefficient $D$ for hydrogen in silica glass is given by Lemaire [17] as

$$
D\left(\mathrm{~cm}^{2} / \mathrm{s}\right)=2.83 \times 10^{-4} \exp \left(-\frac{40.19(\mathrm{~kJ} / \mathrm{mol})}{R T}\right)
$$

where $R=8.31 \mathrm{~J} / \mathrm{K} / \mathrm{mol}$ is the gas constant, and the temperature $T$ is in $K$. The solution on the axis for outward diffusion in cylindrical geometry is conveniently given by Crank [18] as

$$
\frac{C}{C_{0}}=1-\exp \left(-\frac{a^{2}}{4 D t}\right)
$$

where $a$ is the radius of the optical fiber, $62.5 \mu \mathrm{m}$ and $t$ is time after the onset of diffusion from a fully loaded sample.

The experimental data of Fig. 2(b) for an SP-FBG and of Fig. 3 for an LP-FBG are compared with this theory in Fig. 4. The wavelength shifts $\Delta \lambda$ in Figs. 4(a) and (b) are taken with respect to the stable wavelength toward the end of annealing, and data points within about 15 min after annealing have been removed since the oven temperature is not stabilized at those times, as explained previously. For the same reason that the out-diffusion effectively starts after $\sim 15 \mathrm{~min}$, the theoretical curves in Fig. 4(c) are shifted to later times by $15 \mathrm{~min}$. Since the diffusion coefficient $D$ given by (2) is $405,92,26$, and 3.5 times greater than the value at room temperature $\left(22^{\circ} \mathrm{C}\right)$ for annealing temperatures of $200,140,100$, and $50^{\circ} \mathrm{C}$, respectively, the out-diffusion process effectively takes place after the oven temperature has returned to its steady state, and the error caused by the 15-min shift in the theoretical curves should be small. The amount of wavelength shift $\Delta \lambda$ was $\sim 0.7 \mathrm{~nm}$ for the SP-FBG and $\sim 77 \mathrm{~nm}$ for the LP-FBG at any of the annealing temperatures in Fig. 4(a) and (b), respectively. The behaviors of $\Delta \lambda$ for 
(a)

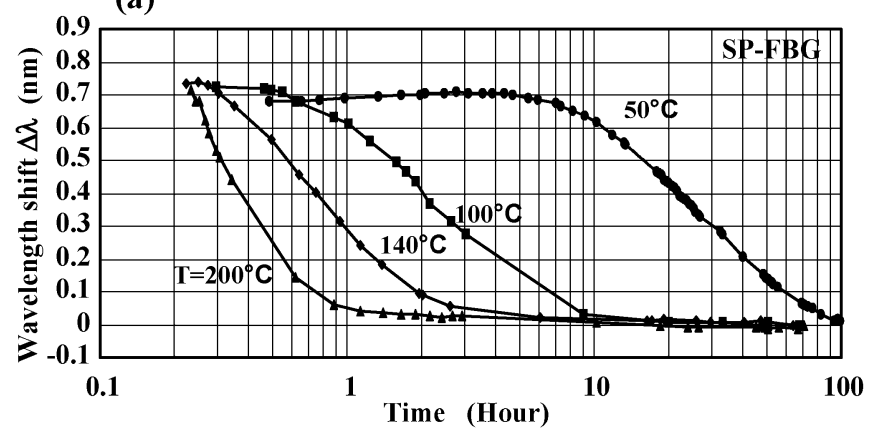

(b)

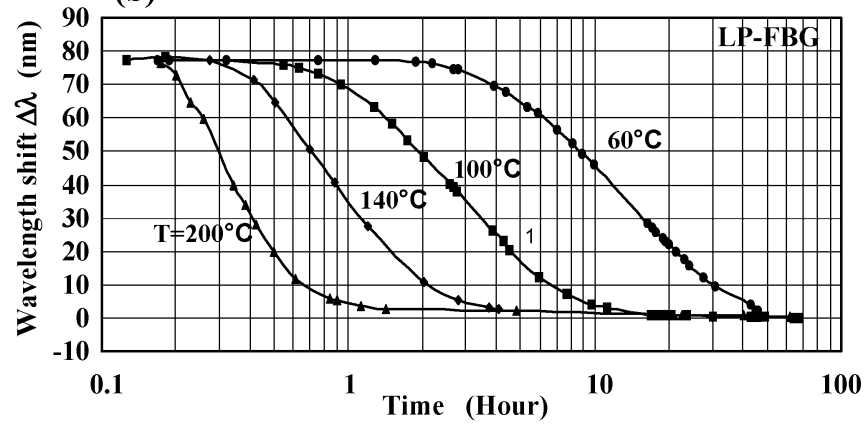

(c)

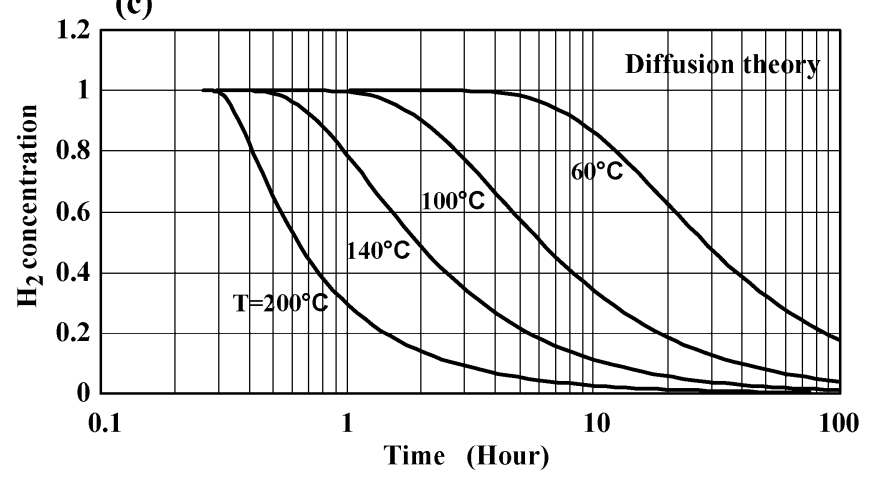

Fig. 4. Evolution of the central wavelength shift $\Delta \lambda$ during annealing for (a) SP-FBG and (b) LP-FBG. Curve (c) is the on-axis hydrogen concentration in the fiber calculated using diffusion theory.

both SP-FBGs and LP-FBGs are nearly identical to Fig. 4(c) for the theoretically expected outward diffusion of hydrogen gas.

\section{Minimum Annealing Time}

From the standpoint of practical applications, it is very useful to know how many hours of annealing are needed to let hydrogen gas diffuse out of an optical fiber in order to achieve a stable central wavelength. The time required to drive out $95 \%$ of the hydrogen, i.e., the time for the wavelength shift to reach $95 \%$ of the total $\Delta \lambda$ is plotted in Fig. 5 as a function of the reciprocal of the annealing temperature. There, the open circles correspond to data for the SP-FBG, and the filled circles correspond to that for the LP-FBG. Both have nearly identical dependences on temperature, and a fitting a curve for both gives

$$
t(h)=2.063 \times 10^{-4} \exp \left(\frac{4079}{T(K)}\right) .
$$

For annealing times longer than this, the hydrogen is nearly all driven out by diffusion from the fiber. Theoretically, this time

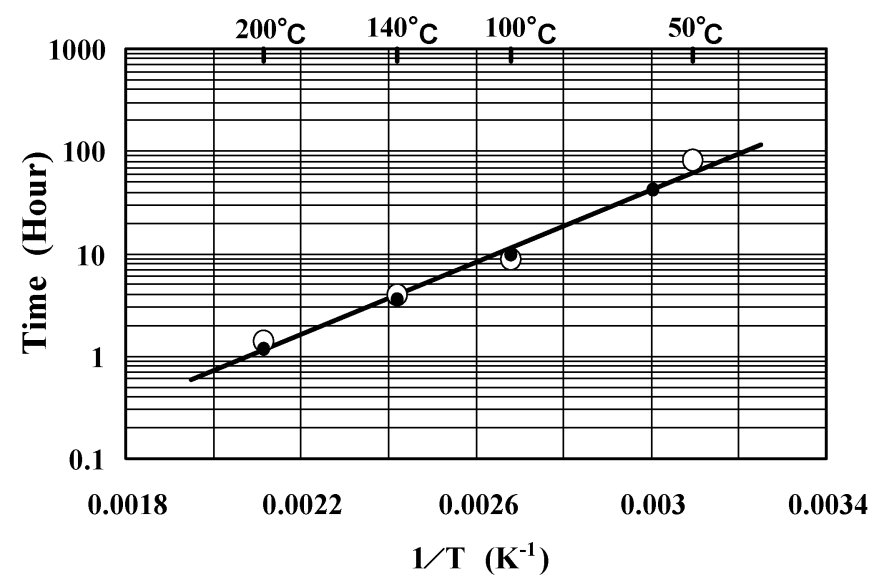

Fig. 5. Annealing time as a function of the reciprocal of the annealing temperature required for stabilization of the central wavelength. Open circles are for SP-FBG, and filled circles are for LP-FBG.

can be calculated by setting (3) equal to $5 \%$. This means that the diffusion coefficient $D$ can be determined experimentally by combining this relation with (4). The resulting coefficient $D$ is

$$
D\left(\mathrm{~cm}^{2} / \mathrm{s}\right)=2.56 \times 10^{-4} \exp \left(-\frac{33.87(\mathrm{~kJ} / \mathrm{mol})}{R T}\right)
$$

in good agreement with (2), Lemaire's formula [17].

\section{E. Dependence of $\Delta \lambda$ on Loading Pressure}

In order to confirm further that the wavelength shift $\Delta \lambda$ of the FBGs is due to diffusion of hydrogen out of the optical fiber, we have conducted experiments in which the hydrogen-loading pressure is changed from 100 to 50 and $10 \mathrm{~atm}$. The resulting evolution of the central wavelength during annealing at $140{ }^{\circ} \mathrm{C}$ is shown in Fig. 6(a) and (b) for SP-FBGs and LP-FBGs, respectively. Data for the case without hydrogen loading are included. In this case, although the transmission minimum was very low at around $-1 \mathrm{~dB}$ because of poor $\mathrm{UV}$ photosensitivity owing to the lack of hydrogen, the spectrum is clearly visible, and the central wavelength did not shift during the entire annealing time. The corresponding theoretical curve is that for $140{ }^{\circ} \mathrm{C}$ in Fig. 4(c). As expected theoretically, the total $\Delta \lambda$ does increase with loading pressure as shown in Fig. 6(c) and (d) for the SP-FBG and LP-FBG, respectively. These curves show some deviation from linearity unlike our expectation of a linear dependence.

\section{F. Change in the Refractive Index Caused by Hydrogen}

Experimental data, especially those in Figs. 4 and 6, demonstrate clearly that the evolution of the central wavelength of FBGs during annealing is defined by the out-diffusion of hydrogen from the optical fiber. The question then arises as to how much the refractive index of the fiber changes as a result of the annealing. For SP-FBGs, this can be analyzed using $\lambda=2 n_{\text {eff }} \Lambda$, and the formula for the modulation $\Delta n_{\text {mod }}$ is given by

$$
1-T=\tanh ^{2}\left(\frac{\Delta n_{\mathrm{mod}}}{n_{\mathrm{eff}}} \frac{\pi L}{2 \Lambda}\right)
$$


(a)

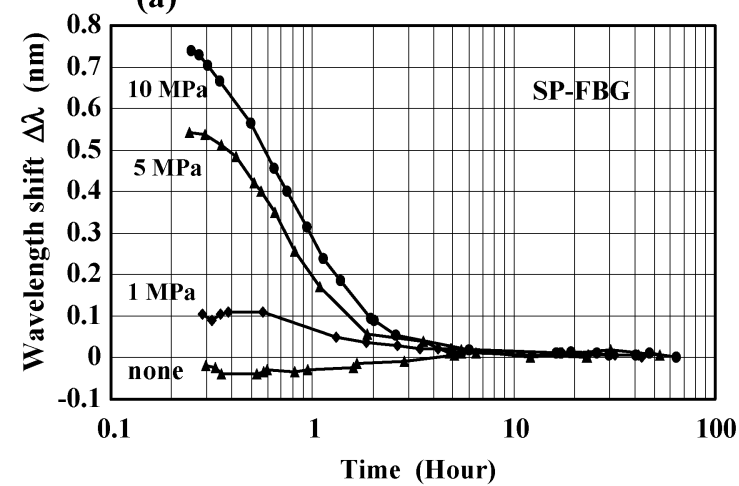

(b)

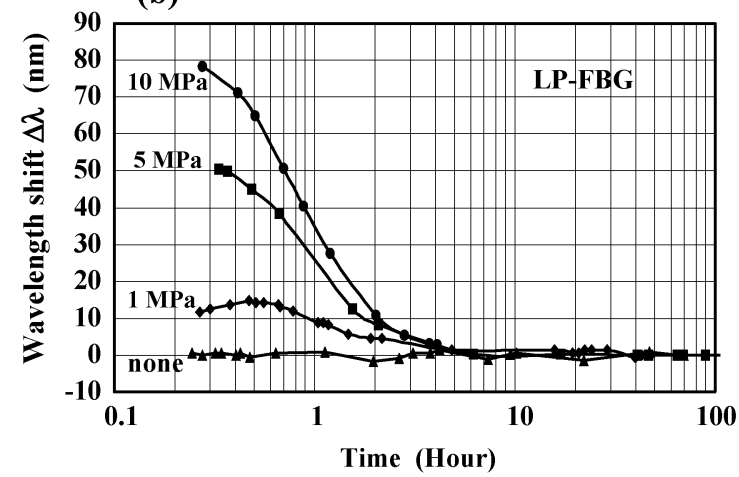

(c)

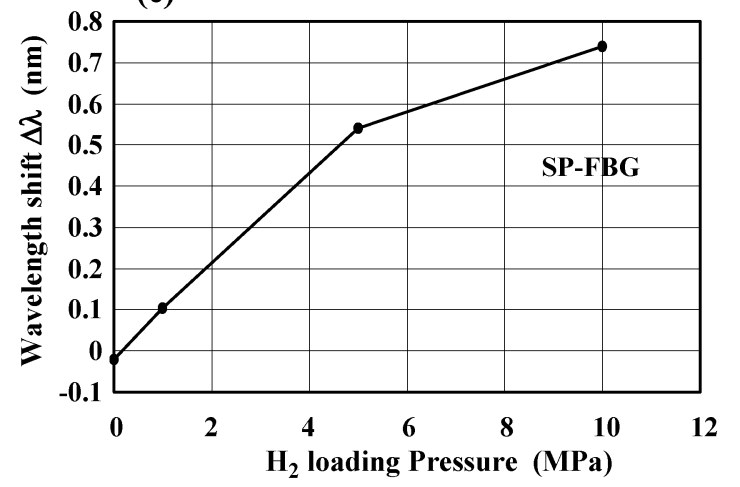

(d)

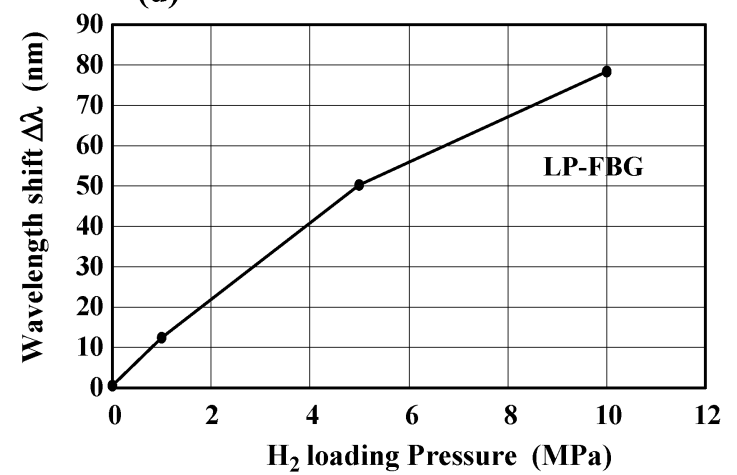

Fig. 6. Variation in the change in the central wavelength $\Delta \lambda$ for different hydrogen-loading pressures: evolution during annealing for (a) SP-FBG and (b) LP-FBG; and (c) and (d) are the total shift $\Delta \lambda$ for SP-FBG and LP-FBG, respectively.

where $T$ is transmission and $L$ the FBG length. The grating pitch $\Lambda$ is half of the phase mask period $p$ which is $1073.63 \mathrm{~nm}$, and $L$ is $15.5 \mathrm{~mm}$. The effective-refractive index $n_{\text {eff }}$ can be calculated from $\lambda=2 n_{\text {eff }} \Lambda$ using the experimentally observed wavelength $\lambda$, and the grating modulation $\Delta n_{\text {mod }}$ can be calculated from (6) using measured transmission $T$. These are summarized in Table I for the case of $140{ }^{\circ} \mathrm{C}$ annealing.

The grating modulation $\Delta n_{\text {mod }}$ contributes to the refractive index by its average value. However, its difference between before and after annealing $\delta\left(\Delta n_{\text {mod }}\right),-0.22 \times 10^{-4}$ is negligibly small compared with the difference of the effective refractive index $\delta\left(n_{\mathrm{eff}}\right),-6.70 \times 10^{-4}$. The fact that the refractive index has decreased by this amount $\delta\left(n_{\mathrm{eff}}\right)$, owing to outward diffusion of hydrogen during annealing, means that the refractive index has already been increased by hydrogen loading to 100 atm before the FBG was fabricated by exposing it to the UV laser. This interpretation suggests that, if an FBG that has been annealed once is reloaded with hydrogen, then the central wavelength $\lambda$ should recover the value it had before the first annealing. In addition, if it is subsequently annealed again, then the $\lambda$ should evolve in the same way as during the first annealing. A reloading experiment of this sort is described next.

\section{G. Reloading Experiment}

In order to test this expectation, we have reloaded hydrogen in an LP-FBG at the same loading pressure for the same number of days and have annealed the sample in the same way as during the first annealing, without exposing it again to the UV laser. An LP-FBG was chosen for this test because it has a much
TABLE I

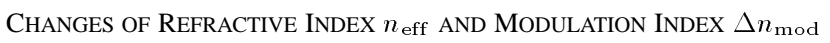
BEFORE AND AFTER THE ANNEALING FOR $140{ }^{\circ} \mathrm{C}$ ANNEALING OF SP-FBG

\begin{tabular}{c|c|c}
\cline { 2 - 3 } & $\begin{array}{c}\text { Before } \\
\text { annealing }\end{array}$ & $\begin{array}{c}\text { After 3 hours } \\
\text { annealing }\end{array}$ \\
\hline \hline Central wavelength $\lambda$ & $1553.25 \mathrm{~nm}$ & $1552.53 \mathrm{~nm}$ \\
\hline Transmission minimum & $-31 \mathrm{~dB}$ & $-25 \mathrm{~dB}$ \\
\hline$n_{\text {eff }}\left(\right.$ from $\left.\lambda=2 n_{\text {eff }} \Lambda\right)$ & 1.44673 & 1.44606 \\
\hline Difference $\delta\left(n_{\text {eff }}\right)$ & --- & $-6.70 \times 10^{-4}$ \\
\hline$\Delta n_{\text {mod }}($ from Eq. $(6))$ & $1.359 \times 10^{-4}$ & $1.138 \times 10^{-4}$ \\
\hline Difference $\delta\left(\Delta n_{\text {mod }}\right)$ & --- & $-0.22 \times 10^{-4}$ \\
\hline
\end{tabular}

larger $\Delta \lambda$ so that it provides better resolution than an SP-FBG. Fig. 7(a) shows the evolution of the central wavelength during the first annealing and Fig. 7(b) the time evolution during a second annealing for two LP-FBGs. The fiber coating resin was removed before exposure to the UV laser and was reapplied after the first annealing. Hydrogen reloading and the second annealing were carried out on the recoated LP-FBG. The time evolution of the central wavelength in the second annealing cycle reproduced that in the first annealing cycle nearly identically, thereby proving that the evolution of the central wavelength is caused entirely by diffusion of hydrogen out of the optical fiber. The first data point in Fig. 7(a) was taken at the end of UV irradiation, and, analogously, the initial data point in Fig. 7(b) was taken before the start of the second annealing cycle. The LP-FBG was recoated within about $2 \mathrm{~h}$ after it was taken out of the oven. In this series of experiments, hydrogen gas was loaded at $90 \mathrm{~atm}$, instead of the $100 \mathrm{~atm}$ used for Fig. 3, and the hydrogen-loaded fiber was processed to UV exposure within a couple of days, instead of the week or two in the case of the 
(a) First annealing

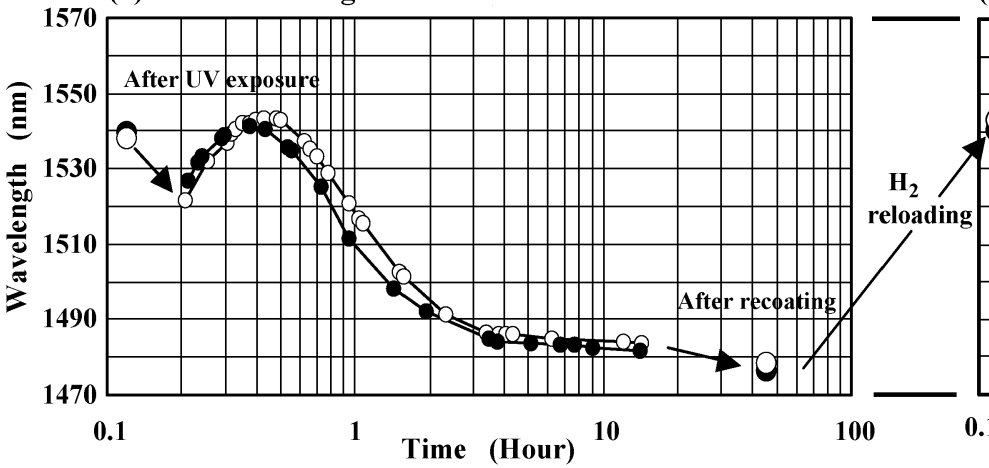

(b) 2nd annealing after $\mathrm{H}_{2}$ reloading

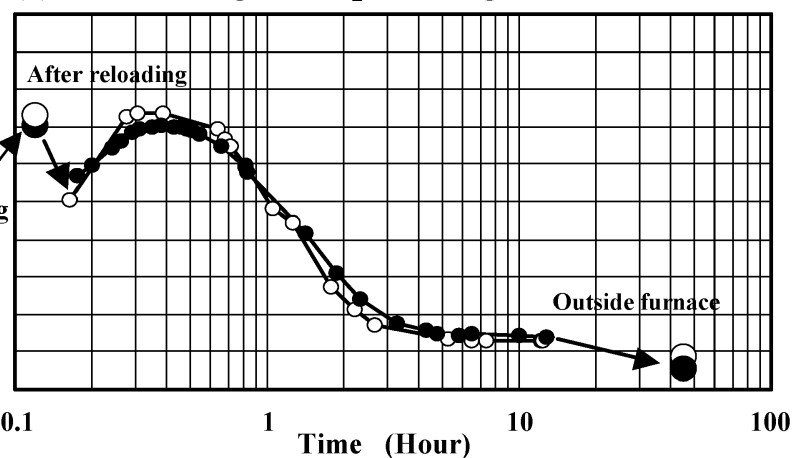

Fig. 7. Evolution of the central wavelength of an LP-FBG with hydrogen reloading: (a) is for the first annealing and (b) for a second annealing after hydrogen reloading.

data of Fig. 3. These differences seem to have yielded a shift of $60 \mathrm{~nm}$, which is less than the 77-nm shift of Fig. 3. The transmission minimum is sensitive to how the fiber has been held in the oven which is indicative of some sensitivity to the weak bending and applied stresses; however, the central wavelength was quite reproducible. The data of Fig. 7 confirm that the evolution of the $\lambda$ is the result of the diffusion of hydrogen out of the fiber.

\section{DISCUSSION}

The physics model of Malo et al. [1] for the depletion of hydrogen molecules by UV laser irradiation followed by in-diffusion of hydrogen to the core of the fiber from surrounding cladding, appears to be very likely. This will cause the central wavelength to increase initially and decrease later. In their experiment, at room temperature, the upswing occurred in the first $4 \sim 5 \mathrm{~h}$. For the annealing temperatures of our study up to $200^{\circ} \mathrm{C}$, it should take place at much earlier times. The time $t_{\text {swing }}$ can be estimated from the simple relation $t_{\text {swing }} \sim x^{2} / D$ using the diffusion coefficient $D$ given by (2) and taking the diffusion path length $x$ as the fiber core diameter $\sim 6 \mu \mathrm{m}$. This crude estimate of the time $t_{\text {swing }}$ for room temperature $20^{\circ} \mathrm{C}$ gives $5 \mathrm{~h}$, in agreement with their experiment. For our annealing temperatures 140 and $200{ }^{\circ} \mathrm{C}$ as in Fig. 2, $t_{\text {swing }}$ is 2.6 and $0.6 \mathrm{~min}$, respectively. Although it is not clear for $50^{\circ} \mathrm{C}$ for which $t_{\text {swing }}$ is $1.1 \mathrm{~h}$, the increases of the wavelength within $10 \sim 15 \mathrm{~min}$ for 140 and $200{ }^{\circ} \mathrm{C}$ seen in Fig. 2 are very likely to be caused by inward diffusion. However, because of uncertainties in the oven temperature in the first 15 min, we do not think our data can resolve the process well.

According to the hypothesis of Malo et al. [1], the wavelength should shift to shorter values in real time during pulsed irradiation with the UV laser as depletion of hydrogen molecules in the fiber core proceeds. The growth of the grating during this stage, i.e., the increase of $\Delta n_{\text {mod }}$, of course, raises background value $n_{\text {eff }}$ by the averaged amount of the modulation, but its magnitude is much smaller than the decrease of $n_{\text {eff }}$ caused by the depletion, probably by the magnitude of the ratio $\delta\left(\Delta n_{\text {mod }}\right)$ to $\delta\left(n_{\text {eff }}\right)$ in Table I which is $0.22 \times 10^{-4} / 6.7 \times 10^{-4} \ll 1$; hence, the shift of the wavelength to shorter values is to be expected. Unlike this expectation, however, we observe a shift to longer wavelengths by the amount of $0.08 \mathrm{~nm}$ momentarily, coincident in time with laser pulse in SP-FBGs, which is caused, we believe, by the temperature increase of the fiber heated by the UV laser pulse [19]. The LP-FBG also did not show the shift. It is not clear to us whether this is due to the opto-lastic effect caused by the release of dilating pressure of dissolved hydrogen molecules.

For an LP-FBG, Bakhti et al. [14] obtained an upswing of the central wavelength very similar to that in SP-FBG by Malo et $a l$. [1], but for a different reason. The central wavelength of an LP-FBG is given by

$$
\lambda=\Lambda\left(n_{\mathrm{eff}}^{01}-n_{\mathrm{eff}}^{0 m}\right)
$$

where $n_{\mathrm{eff}}^{01}$ and $n_{\mathrm{eff}}^{0 m}$ are the effective refractive indexes of the fundamental mode $\mathrm{LP}_{01}$ and cladding mode $\mathrm{LP}_{0 m}$, respectively. The index $n_{\mathrm{eff}}^{0 m}$ is defined by the cladding. When hydrogen out-diffuses from the outer portion of the fiber, i.e., the cladding $n_{\mathrm{eff}}^{0 m}$ decreases and, consequently, $\lambda$ increases. As the hydrogen continues to diffuse out from the bulk of fiber, both $n_{\text {eff }}^{01}$ and $n_{\text {eff }}^{0 m}$ decrease and $\lambda$ decreases, thus giving an upswing in $\lambda$. This interpretation predicts larger upswing for uniformly loaded fiber and a weaker one for partially out-diffused fiber. Our data in Fig. 3 do not show any upswing, but the data for hydrogen reloading in Fig. 7 appear to show an upswing both after UV exposure and after reloading. The data in Fig. 3 have been taken using fibers stored in a freezer for about a week at about $-50^{\circ} \mathrm{C}$ after hydrogen loading, whereas the data in Fig. 7 were taken within a couple of days. Therefore the LP-FBG of Fig. 7 had hydrogen more uniformly loaded over the fiber cross section than that of Fig. 3, and their interpretation regarding the upswing in $\lambda$ seems to apply to our data qualitatively.

Although our annealing data for the evolution of central FBG wavelength have some uncertainties early in time for about 15 minutes because of increasing oven temperature and the initial upswing as described above, the majority of data extending from 15 minutes to 100 hours are well described by the out-diffusion theory for both SP-FBG's and LP-FBG's.

Recently an interesting experiment was done by Viswanathan et al. [20] showing that hydrogen loading can be done using dilute hydrogen gas mixed with other gases such as $\mathrm{Ar}, \mathrm{CO}_{2}, \mathrm{CH}_{4}$ 
and $\mathrm{C}_{2} \mathrm{H}_{6}$. In their experiment, they have shown that the refractive index increases linearly with loading hydrogen pressure. In our experiment, as shown in Fig. 6(c) and (d), the dependence deviated from linearity unlike their result. This difference is not well understood at the moment.

Several recent reports show that the UV photo sensitivity can be enhanced by blank exposure to UV laser light that is, pre-sensitization [21]-[23]. Effective imprinting of FBG at later times has been demonstrated in such fibers with weaker degradation of the transmission minimum during high temperature annealing than in the case of hydrogen loaded fiber. They also manifest little shift of the central wavelength, typically less than $0.1 \mathrm{~nm}$, because of the absence of hydrogen gas. Ref. [23] shows that pre-sensitization can be effective even with a cheap UV lamp. This is certainly very attractive for FBG fabrication when the offset in the central wavelength does not have to be predicted.

\section{CONCLUSION}

The evolution of the central wavelength of FBGs during annealing has been experimentally proven to be caused by diffusion of hydrogen gas out of the optical fiber, which has been loaded with hydrogen before being exposed to UV laser light. The time evolution has been examined for both SP-FBG and LP-FBG. When an FBG is reloaded with hydrogen and reannealed, but without re-exposure to a UV laser, the evolution of the central wavelength during re-annealing is the same as that seen in the first annealing; therefore, the evolution is clearly determined by the diffusion of hydrogen out of the optical fiber.

A formula (4) for the minimum number of hours of annealing required to drive the hydrogen out of the fiber that is consistent with theoretical model of hydrogen out-diffusion has been derived.

\section{ACKNOWLEDGMENT}

The authors would like to thank the referee for kind advice pointing out two articles [14] and [20] of which the authors were not aware at the time of manuscript submission. The authors also wish to thank Prof. K. Saito of Toyota Institute of Technology for his suggestion of reloading the experiment (Fig. 7) for stronger evidence of hydrogen out-diffusion from optical fiber. Finally, the authors also thank Dr. D. H. McNeill for his careful proofreading of the manuscript.

\section{REFERENCES}

[1] B. Malo, J. Albert, K. O. Hill, F. Bilodeau, and D. C. Johnson, "Effective index drift from molecular hydrogen diffusion in hydrogen-loaded optical fibers and its effect on Bragg grating fabrication," Electron. Lett., vol. 30, no. 5, pp. 442-444, 1994.

[2] L. Dong, J. L. Archambault, L. Reekie, P. St. J. Russel, and D. N. Payne, "Photoinduced absorption change in germanosilicate preforms: Evidence for the color-center model of photosensitivity," Appl. Opt., vol. 34, no. 18, pp. 3436-3440, 1995.

[3] H. G. Limberger, P. Y. Fonjallaz, R. P. Salathe, and F. Cochet, "Compaction- and photoelastic-induced index changes in fiber Bragg gratings," Appl. Phys. Lett., vol. 68, no. 22, pp. 3069-3071, 1996.
[4] M. Douay, W. X. Xie, T. Taunay, P. Bernage, P. Niay, P. Cordier, B. Poumellec, L. Dong, J. F. Bayon, H. Poignant, and E. Delevaque, "Densification involved in the UV-based photosensitivity of silica glasses and optical fibers," J. Lightwave Technol., vol. 15, pp. 1329-1342, Aug. 1997.

[5] P. J. Lemaire, R. M. Atkins, V. Mizrahi, and W. A. Reed, "High pressure $\mathrm{H}_{2}$ loading as a technique for achieving ultrahigh UV photosensitivity and thermal sensitivity in $\mathrm{GeO}_{2}$ doped optical fibers," Electron. Lett., vol. 29, no. 13, pp. 1991-1993, 1993.

[6] T. Erdogan, V. Mizrahi, P. J. Lemaire, and D. Monroe, "Decay of ultraviolet-induced fiber Bragg gratings," J. Appl. Phys., vol. 76, no. 1, pp. 73-80, 1994.

[7] S. R. Baker, H. N. Rourke, V. Baker, and D. Goodchild, "Thermal decay of fiber Bragg gratings written in boron and germanium codoped silica fiber," J. Lightwave Technol., vol. 15, pp. 1470-1477, Aug. 1997.

[8] S. Pal, J. Mandal, T. Sun, and T. V. Grattan, "Analysis of thermal decay and prediction of operational lifetime for a type I boron-germanium codoped fiber Bragg grating," Appl. Opt., vol. 42, no. 12, pp. 2188-2197, 2003

[9] G. Brambilla and H. Rutt, "Fiber Bragg gratings with enhanced thermal stability," Appl. Phys. Lett., vol. 80, no. 18, pp. 3259-3261, 2002.

[10] M. Fokine, "Formation of thermally stable chemical composition gratings in optical fibers," J. Opt. Soc. Amer. B, vol. 19, no. 8, pp. 1759-1765, 2002.

[11] L. B. Fu, G. Tan, W. J. Xu, H. L. An, X. M. Cui, X. Z. Lin, and H. D. Liu, "Ultraviolet-initiated reactions of $\mathrm{H}_{2}$ with germanosilicate fibers and $\mathrm{H}_{2}$ concentration dependence of the Bragg wavelength of a fiber grating," Opt. Lett., vol. 25, no. 8, pp. 527-529, 2000

[12] P. L. Swart and A. A. Chtcherbakov, "Study of hydrogen diffusion in boron/germanium codoped optical fiber," J. Lighwave Technol., vol. 20, pp. 1933-1941, Nov. 2002.

[13] P. L. Swart, A. A. Chtcherbakov, W. L. Jourbert, and M. G. Shlyagin, "Study of the pressure dependence of hydrogen diffusion in optical fiber by an interferometric technique," Opt. Commun., vol. 217, pp. 189-196, 2003.

[14] F. Bakhti, J. Larrey, P. Sansonetti, and B. Poumellec, "Impact of hydrogen in-fiber and out-fiber diffusion on central wavelength of UV-written long period gratings," in OSA Tech. Dig. 1997 Topical Meeting Bragg Gratings, Photosensitivity and Poling in Glass Fibers and Waveguides: Fundamentals and Applications, vol. 17, Washington DC, 1997, OSA Tech. Series, Paper BsuD4-1, pp. 55-57.

[15] K. Nakagawa, Y. Takemura, R. Kunimoto, Y. Mizutani, S. Kimura, Y. Fukuyama, Y. Suzaki, and S. Ejima, "Fabrication of fiber gratings with different Bragg wavelengths using a single phase mask," Jpn. J. Appl. Phys., pt. 2, vol. 41, no. 5B, pp. L599-601, 2002.

[16] A. M. Vengsarker, J. R. Pedrazzani, J. B. Judkins, P. J. Lemaire, N. S. Bergano, and C. R. Davidson, "Long-period fiber-grating as band-rejection filters," Opt. Lett., vol. 21, no. 5, pp. 336-338, 1996.

[17] P. J. Lemaire, "Reliability of optical fibers exposed to hydrogen: Prediction of long-term loss increases," Opt. Eng., vol. 30, no. 6, pp. 780-789, 1991.

[18] J. Crank, The Mathematics of Diffusion. Oxford, U.K.: ClarendonPress, 1975, p. 30.

[19] M. Nakamura, C. Komatsu, Y. Masuda, K. Fujita, M. Yamauchi, Y. Mizutani, S. Kimura, Y. Suzaki, T. Yokouchi, K. Nakagawa, and S. Ejima, "Evolution of optical fiber temperature during fiber Bragg grating fabrication using $\mathrm{KrF}$ excimer laser," Jpn. J. Appl. Phys., vol. 43, no. 1, pp. 147-151, 2004.

[20] N. K. Viswanathan, W. V. Dower, D. M. Paolucci, and M. D. Barrera, "Enhancing fiber photosensitivity using dilute hydrogen in high-pressure mixtures," in OSA Tech. Dig. 2003 Topical Meeting Bragg Gratings, Photosensitivity and Poling in Glass Fibers and Waveguides: Fundamentals and Applications, Washington DC, 2003, OSA Tech. Series, Paper MC5, pp. 49-52.

[21] M. Aslund and J. Canning, "Annealing properties of gratings written into UV-presensitized hydrogen-outdiffused optical fiber," Opt. Lett., vol. 25, no. 10, pp. 692-694, 2000.

[22] M. Lancry, P. Niay, S. Bailleux, M. Douay, C. Depecker, P. Cordier, and I. Riant, "Thermal stability of the 248-nm-induced presensitization process in standard $\mathrm{H}_{2}$-loaded germanosilicate fibers," Appl. Opt., vol. 41, no. 34, pp. 7197-7204, 2002.

[23] A. Canagasabey and J. Canning, "UV lamp hypersensitization of hydrogen-loaded optical fibres," Opt. Express, vol. 11, no. 13, pp. $1585-1589,2003$. 


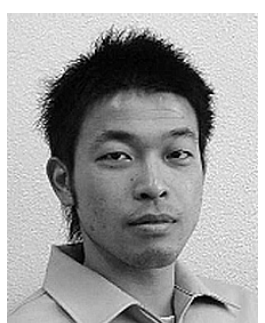

Yuji Masuda received the Bachelor's degree in applied materials science from the Faculty of Engineering, Kagawa University, Kagawa, Japan, in 2003. He is currently working toward the M.Sc degree at the same university.

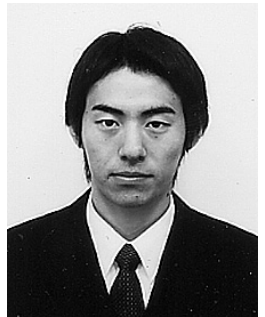

Mitsuo Nakamura received the Bachelor's degree in applied materials science from the Faculty of Engineering, Kagawa University, Kagawa, Japan, in 2003. He is currently working toward the M.Sc. degree in materials science at the Graduate School of Engineering, Osaka University, Osaka, Japan.

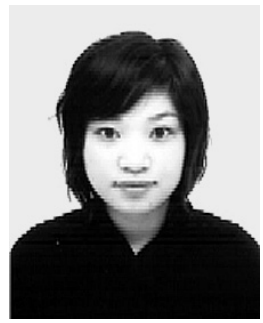

Chisa Komatsu received the Bachelor's degree in applied materials science from the Faculty of Engineering, Kagawa University, Kagawa, Japan, in 2003.

She currently works for Fujitsu Kochi Systems Engineering, Ltd., Kochi, Japan.

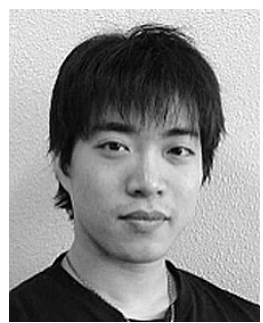

Keio Fujita received the Bachelor's degree in applied materials science from the Faculty of Engineering, Kagawa University, Kagawa, Japan, in 2003. He is currently working toward the M.Sc. degree at the same university.

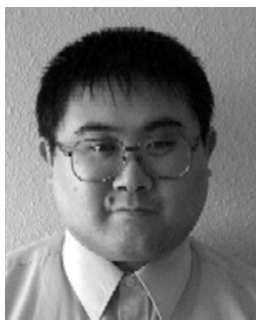

Makoto Yamauchi received the Bachelor's degree in intelligent mechanical systems engineering from the Faculty of Engineering, Kagawa University, Kagawa, Japan, in 2002.

He currently works for Shinko Electric Wire Company, Ltd., Kagawa, Japan.

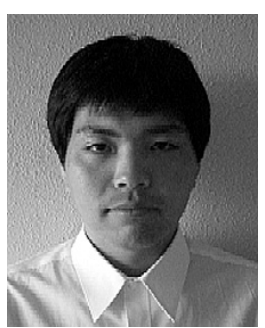

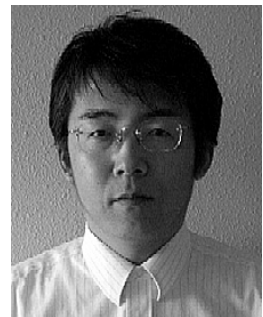

Yasuo Mizutani received the Bachelor's degree in applied physics from the Engineering Department, Tokai University, Kanagawa, Japan, in 1986.

He has worked for Shinko Electric Wire Company, Ltd., Kagawa, Japan, since 1986.

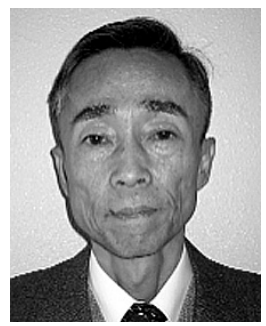

Susumu Kimura received the Bachelor's degree in applied chemistry from the Engineering Department, Yokohama National University, Yokohama, Japan, in 1972.

$\mathrm{He}$ is Director of the Technology Division of Shinko Electric Wire Company, Ltd., Kagawa, Japan.

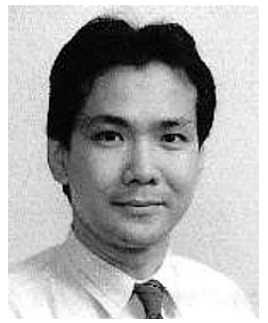

Yoshifumi Suzaki (M'94) received the Bachelor's degree, the M.Sc. degree in precision engineering, and the Dr.Eng. degree from Osaka University, Osaka, Japan, in 1985, 1987, and 1995, respectively.

$\mathrm{He}$ was Associate Professor at Takamatsu National College of Technology from 1987 to 2000. Since 2000, he has been an Associate Professor in the Faculty of Engineering, Kagawa University, Kagawa, Japan.

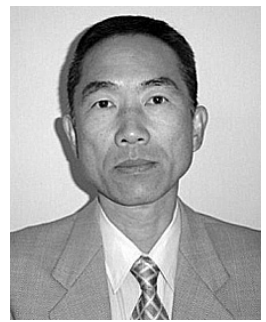

Takashi Yokouchi received the Bachelor's degree in law from the Law Department, Hosei University, Tokyo, Japan, in 1986

He is currently on the technical staff of the Faculty of Engineering, Kagawa University, Kagawa, Japan.

Kiyoshi Nakagawa (S'69-M'82-SM'91) received the M.Sc. degree in law from the Graduate School of Science and Technology, Kobe University, Kobe, Japan, in 1986.

He has been an Associate Professor at the Faculty of Engineering, Kagawa University, Kagawa, Japan, since 2001.

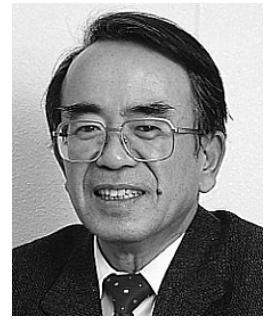

Seiki Ejima received the M.Sc. degree in telecommunications from Waseda University, Tokyo, Japan, in 1970 and the Ph.D. degree in plasma physics from Columbia University, New York, in 1973.

He worked on nuclear fusion research from 1974 to 1987 at General Atomic Company, San Diego, CA, and on electronics material research and development at Shin-Etsu Chemicals Company, Ltd., from 1987 to 2000. Since 2000, he has been a Professor in the Faculty of Engineering, Kagawa University, Kagawa, Japan. 